
\title{
3 Research Square on \\ Dramatic Activation of an Antibody by a Single Amino Acid Change in Framework
}

\section{Wei-Ching Liang}

Genentech Inc

Jianping Yin

Genentech Inc

Patrick Lupardus

Genentech Inc

Jianhuan Zhang

Genentech Inc

Kelly M. Loyet

Genentech Inc

Jawahar Sudhamsu

Genentech Inc

Yan Wu ( $\nabla$ yw@gene.com )

Genentech Inc

\section{Research Article}

Keywords: Humanization, Complementarity-determining regions (CDRs), Fab-Fab interaction, allosteric blocking, antigen/antibody complex crystal structures

Posted Date: September 7th, 2021

DOI: https://doi.org/10.21203/rs.3.rs-862975/v1

License: (c) (i) This work is licensed under a Creative Commons Attribution 4.0 International License.

Read Full License

Version of Record: A version of this preprint was published at Scientific Reports on November 16th, 2021. See the published version at https://doi.org/10.1038/s41598-021-01530-w. 


\section{Abstract}

Antibody function is typically entirely dictated by the Complementarity Determining Regions (CDRs) that directly bind to the antigen, while the framework region acts as a scaffold for the CDRs and maintains overall structure of the variable domain. We recently reported that the rabbit monoclonal antibody $4 \mathrm{~A} 11$ (rbt4A11) disrupts signaling through both TGF $\beta 2$ and TGF $\beta 3^{1}$. Here, we report a dramatic, unexpected discovery during the humanization of rbt4A11 where, two variants of humanized $4 \mathrm{~A} 11$ (h4A11), v2 and v7 had identical CDRs, maintained high affinity binding to TGF $32 / 3$, yet exhibited distinct differences in activity. While h4A11.v7 completely inhibited TGF $32 / 3$ signaling like rbt4A11, h4A11.v2 did not. We solved crystal structures of TGF 32 complexed with Fab fragments of h4A11.v2 or h4A11.v7 and identified a novel interaction between the two heavy chain molecules in the 2:2 TGFb2:h4A11.v2-Fab complex. Further characterization revealed that framework residue variations at either position 19,79 or 81 of the heavy chain strikingly converts h4A11.v2 into an inhibitory antibody. Our work suggests that in addition to CDRs, framework residues and interactions between Fabs in an antibody could be engineered to further modulate activity of antibodies.

\section{Introduction}

Transforming Growth Factor-beta (TGF $\beta$ ) is a key driver of fibrogenesis. There are three TGF $\beta$ isoforms with highly homologous receptor-binding domains with high sequence similarity, and have similar effects on target cells in vitro. Unlike TGF $\beta 1$, both TGF 32 and TGF $\beta 3$ are expressed at elevated levels in human lung and liver fibrotic tissues and can be activated via distinct mechanisms ${ }^{1}$. Further, inhibiting both TGF $\beta 2$ and TGF $\beta 3$ while sparing TGF $\beta 1$ could alleviate lung fibrosis while avoiding toxicity concerns associated with pan-TGF $\beta$ blockade. We recently identified an isoform-selective rabbit monoclonal antibody (rbt4A11) that binds mature forms of both TGF 32 and TGF 33 with picomolar (pM) affinity, but shows no binding to the mature form of TGF $\beta 1$. It also has potent inhibitory activity against both TGF $\beta 2$ and TGF 33 with the potential to be developed as a therapeutic for lung fibrosis. Humanization of rbt4A11 was performed to enable development of a potential clinical candidate for non-human primate pharmacokinetics and safety studies. Unlike previously described pan-TGF $\beta$ blocking antibodies such as Fresolimumab (Sanofi), the structure of a humanized rbt4A11 Fab (h4A11.v7) complexed with TGFß2 shows that the epitope for h4A11.v7 is not near the TGF $\beta R 1 / R 2$ binding site, and it does not sterically compete for receptor binding. Instead h4A11.v7 Fabs induce a conformational change in TGF $\beta 2$, causing the two monomers to "pinch" together by several degrees, demonstrating an allosteric mechanism of inhibition ${ }^{1}$.

For humanization of rbt4A11, we adopted well-accepted approaches in humanization of rodent (mouse and rat) monoclonal antibodies (mAbs) by grafting complementarity-determining regions (CDRs) and framework residues at the Vernier zone of rabbit antibody onto the human immunoglobulin germline gene segments which shared the highest sequence identity ${ }^{2,3}$. Combining this approach with the framework shuffling strategy, an efficient and effective way to manipulate and improve antibody 
properties in humanization of murine mAbs allowed us to rapidly select the most favorable combinations

of human germline frameworks to maintain the affinity and activity of the parental antibody ${ }^{4,5}$.

In the course of rbt4A11 humanization, the entire panel of humanized 4A11 (h4A11) variants exhibited pM binding affinities very similar to the parental clone, but surprisingly varied in inhibition properties for TGF $\beta 2$ and TGF $\beta 3$ in a cell-based activity assay. To better understand the molecular mechanisms that conferred drastically different functionality of the different humanized variants that still had almost identical CDRs, we solved crystal structures of antigen-binding fragments (Fabs) from a complete blocker (h4A11.v7)(previous work) ${ }^{1}$ and an incomplete blocker (h4A11.v2)(current work) in complex with human TGF $\beta 2$. The structures revealed specific interactions between framework regions in the heavy chains of the two h4A11.v2 Fabs bound to the TGF 32 dimer. Disruption of this interaction by a single amino acid variation at either position 19,79 or 81 in the heavy chain framework converts h4A11.v2 to a 'complete blocker', similar to h4A11.v7 and rbt4A11.

\section{Methods}

\section{Humanization, IgG variants cloning, expression and purification}

The variable regions of rabbit antibody 4A11 (rbt4A11) amino acid sequence were aligned to its closest human germlines using informatics tool (AbGrafter, Genentech) which covered all available immunoglobulin IGKV and IGHV mammalian human germline genes (Homo sapiens) in the international immunogenetics information system ${ }^{10}$. The closest human IGKV and IGHV germline frameworks identified through the process were then served as the acceptor frameworks for grafting rbt4A11 light chain and heavy chain hypervariable regions with the corresponding rabbit framework residues at the Vernier zone respectively ${ }^{3}$. By mixing and matching these grafting acceptor frameworks, a total combination of eight humanized 4A11 variants (h4A11.v1 - h4A11.v8) were generated.

The coding sequences of each humanized variant's variable region were generated by DNA synthesis and cloned into human IgG1 expression vectors (Genewiz). Small-scale IgG expressions and purifications were done in a high-throughput format. Briefly, IgG expressions were transiently conducted in Expi293F cells (Thermal Fisher Scientific), followed by a 2-step purification using Protein A affinity chromatography (MabSelect SuRe ${ }^{\mathrm{T}}{ }^{\mathrm{N}}$, Cytiva) and analytical size exclusion chromatography (SEC, Cytiva). Quality control of antibody's purity was determined by SDS-PAGE with Coomassie blue staining, A280 absorbance to measure protein concentration and aggregation analysis by SEC, as previously described $^{11,12}$.

\section{Expression, purification and crystallization of human TGFB2 and h4A11.v2 /h4A11.v7 Fab}

The mature form of human TGF 32 was produced as previously reported ${ }^{13}$. LAP-TGF $\beta 2$ was expressed with a $\mathrm{CHO}$ TI stable cell line. Protein was purified with Ni-NTA, then was acidified and dialyzed in $0.1 \mathrm{M}$ sodium citrate, $\mathrm{pH} 3$ and loaded onto a $5 \mathrm{ml} \mathrm{SP} \mathrm{HP}$ column. The fractions that contained the 
protein of interest from the SP pool were then further purified with Vydac C4 RP-HPLC. The fraction containing mature TGF $\beta 2$ was dialyzed directly into $1 \mathrm{mM} \mathrm{HCl} \mathrm{pH} \mathrm{3.0.}$

The Fab fragments of anti-TGFB2 (h4A11.v2 and h4A11.v7)were expressed in E. coli overnight at $30^{\circ} \mathrm{C}$ under the control of the PhoA promoter. The cells were pelleted by centrifugation at $6000 \mathrm{rpm}$ for 15 minutes and lysed by microfluidization in PBS supplemented with 25 mM EDTA and 1mM PMSF. Cell debris was removed by centrifugation at $10,000 \mathrm{rpm}$ for 1 hour at $4^{\circ} \mathrm{C}$. The resulting supernatant was run through a Protein $\mathrm{G}$ column equilibrated in PBS, and eluted with $0.58 \%$ acetic acid. Protein fractions were further purified by ion exchange chromatography (SP-sepharose) in $20 \mathrm{mM} \mathrm{MES} \mathrm{(pH} \mathrm{5.5)} \mathrm{and} \mathrm{eluted} \mathrm{with}$ a gradient from 0 to $250 \mathrm{mM} \mathrm{NaCl}$.

The TGF 32 and h4A11.v2 Fab complex was mixed at 1:1 molar ratio and further purified using a Superdex-200 column equilibrated in $25 \mathrm{mM}$ Tris- $\mathrm{HCl}(\mathrm{pH}$ 7.5) and $200 \mathrm{mM} \mathrm{NaCl}$. For crystallization, the complex samples were concentrated to $10 \mathrm{mg} / \mathrm{ml}$. The h4A11.v2 Fab/TGF $\beta 2$ crystals were obtained by vapor diffusion at $19^{\circ} \mathrm{C}$ with mixing equal volumes of protein plus $15 \%$ PEG 4000 and $0.1 \mathrm{M} \mathrm{Na}$ Cacodylate pH 6.0 well solution. Crystals were cryoprotected with $25 \%$ glycerol. Data set was collected at the Advanced Light Source beamline 5.0.2 and processed with the HKL package ${ }^{14}$. Crystal structures were solved by molecular replacement using Phaser ${ }^{15}$. The refined coordinates of the TGFß2 structure PDB file (619J) served as the search probe for the structure for TGF $\beta 2^{16}$. Subsequently, the constant (Fc) and variable (Fv) portions of the Fab were placed separately using Phaser and underwent initial rounds of rigid-body refinement with Phenix ${ }^{17}$. The model went through several iterative rounds of adjustment with $\operatorname{Coot}^{18}$. Atomic models were then built and refined with Phenix ${ }^{17}$.

\section{TGF $\beta$ binding affinity measurement}

The binding affinity of each humanized 4A11 (h4A11) variant was determined by surface plasmon resonance (SPR) technology (Biacore ${ }^{\mathrm{TM}}-\mathrm{T} 200$, Cytiva). Briefly, Series S sensor chip Protein A (Cytiva) was utilized to capture each version of h4A11 antibody on a different flow cell (FC) to achieve approximately 90 response units $(\mathrm{RU})$, followed by the injection of five-fold serial dilutions of each human mature TGF $\beta$ isoform (PeproTech; $0.008 \mathrm{nM}$ to $5 \mathrm{nM}$ ) in HBS-EP buffer (100 mM 4-(2-hydroxyethyl)-1piperazineethanesulfonic acid (HEPES) pH 7.4, $150 \mathrm{mM} \mathrm{NaCl}, 3 \mathrm{mM}$ EDTA, 0.05\% (v/v) Surfactant P20) with a flow rate of $100 \mu \mathrm{l} / \mathrm{min}$ at $37^{\circ} \mathrm{C}$ to reach a relative low maximum binding response (Rmax) around $30 \mathrm{RU}$. The association was monitored by a standard $3 \mathrm{~min}$ observation, whereas the dissociation was extended to a much longer time (60 mins) to receive sufficient decay for accurate off-rate measurement. The sensorgrams were recorded, processed by reference and blank subtraction, and evaluated by a simple one-to-one Langmuir binding model (Biacore T200 evaluation software version 2.0) to determine the equilibrium dissociation constant $\left(K_{D}\right)$.

\section{TGF $\beta$ inhibition cell-based assay}


The blocking potency of each h4A11 variant against each human mature TGF $\beta$ isoform was evaluated by a HEK-Blue ${ }^{\text {TM }}$ TGF $\beta$ cell-based reporter assay. HEK-Blue ${ }^{\text {TM }}$ TGF $\beta$ cells (InvivoGen) were generated by stable transfection of HEK293 cells with the human TGF $\beta$ receptor 1, Smad3, and Smad4 genes, and further expressed a Smad3/4-binding elements (SBE)-inducible secreted embryonic alkaline phosphatase (SEAP) reporter gene. Before experiments, cells were maintained in DMEM high glucose medium containing $10 \%$ fetal bovine serum (VWR), $2 \mathrm{mM} \mathrm{L-glutamine,} 50$ units $/ \mathrm{mL}$ penicillin, $50 \mu \mathrm{g} / \mathrm{mL}$ streptomycin, $100 \mu \mathrm{g} / \mathrm{ml}$ Normocin ${ }^{\mathrm{TM}}, 30 \mu \mathrm{g} / \mathrm{ml}$ of blasticidin, $200 \mu \mathrm{g} / \mathrm{ml}$ of Hygromycin B Gold and 100 $\mu \mathrm{g} / \mathrm{ml}$ of Zeocin ${ }^{\text {TM }}$ (InvivoGen) at $37^{\circ} \mathrm{C} \pm 0.5^{\circ} \mathrm{C}$ with $5 \% \mathrm{CO}_{2}$. On the assay day, cells were seeded in cells were seeded in Greiner Bio-One ${ }^{T M}$ CellStar ${ }^{\text {TM }}$ 384-Well, cell culture-treated, flat-bottom microplates at a density of 9000 cells per well in the test medium (DMEM high glucose supplemented with $10 \%$ heat inactivated fetal bovine serum, 50 units $/ \mathrm{mL}$ penicillin, $50 \mu \mathrm{g} / \mathrm{mL}$ streptomycin, and $2 \mathrm{mM}$ glutamine) and incubated for 15 mins at the condition described above. Each antibody variant, 4-fold serially diluted for 12 points (final $0.000159 \mathrm{nM}$ to $667 \mathrm{nM}$ ), was incubated with each human mature TGF $\beta$ isoform (PeproTech; final $20 \mathrm{pM}$ ) for $1 \mathrm{hr}$, followed by transferring to the plates containing cells. The SEAP in the supernatant was measured with Quanti-Blue (InvivoGen) per manufacturer's instructions after $37^{\circ} \mathrm{C}$ incubation for 18-22 hrs. All experiments were done in quadruplicates, and the maximum or minimum response was determined by TGF $\beta$ or test media only respectively for normalization. The half maximal inhibitory concentration $\left(\mathrm{IC}_{50}\right)$ values were then calculated by fitting the titration curves ([Antibody variant] vs. \% Maximum TGF $\beta$ activity) using a Prism variable slope four parameters model (GraphPad Software).

\section{Results}

\section{Humanization of rabbit mAb $4 A 11$ by grafting $C D R s$ to the closest human frameworks}

Rabbit monoclonal antibody 4A11 (rbt4A11) was humanized in a two-step strategy. First, the human germline frameworks that are closest to the variable light chain $(\mathrm{VL})$ and variable heavy chain $\mathrm{VH})$ of rbt4A11 were identified based on the selection criteria of the highest amino acid sequence identity in variable region, frequent usage in antibody repertoire, and functional genes in different germline family. In total, we found four human light chain germline genes (IGKV1D-39*01, IGKV4-1*01, IGKV3-20*01, IGKV2-24*01) and two human heavy chain germline genes (IGHV3-48*01, IGHV4-59*06) as the closest acceptor frameworks, which were $62 \%, 61 \%, 54 \%$ and $54 \%$ identical to the VL, and $57 \%$ and $52 \%$ identical to the $\mathrm{VH}$ of rbt4A11, respectively. Subsequently, the rbt4A11 CDRs, which covered the definition of Kabat

and Chothia ${ }^{6}$, and fifteen rabbit framework residues at the Vernier zone (position 2, 4, 36, 43 and 58 of VL, and position 2, 24, 37, 48, 49, 67, 71, 78, 91 and 105 of $\mathrm{VH}$ ) were grafted onto each acceptor framework to generate four humanized VL and two humanized VHgene segments for molecular cloning (Figure 1). To assess the best humanized variants, a panel of eight humanized 4A11 variants (h4A11.v1 - h4A11.v8) was generated through mixing and matching the cloned humanized gene segments and expressing each variant as human IgG1 for further characterization (Table 1). The expression and purification profiles of all humanized variants were good ( $>99 \%$ monomer) with no noticeable differences between germline 
usage. The variable domains of these eight humanized $4 A 11$ variants comprised an identity of $77 \%$ to $81 \%$ in the human immunoglobulin germline sequence, which were further improved by framework Vernier permutation from original rabbit residues to the corresponding human germline residues to meet World Health Organization standards as a humanized antibody (data not shown).

\section{Functional characterization of humanized $4 \mathrm{~A} 11$ variants}

Each humanized 4A11 variant was purified and characterized by measuring its binding affinity and blocking activity against all three mature TGF $\beta$ isoforms. To minimize the avidity effect on binding, the affinity of each variant was measured by real-time Biacore SPR using label-free TGF $\beta$ in solution with low level of antibody bound to the biosensor surface, as previously described ${ }^{6}$. Further, the blocking activity of the variant IgGs to inhibit mature TGF $\beta$ induced TGF $\beta$ receptor dependent signaling was determined using reporter cell lines (HEK-Blue ${ }^{\mathrm{TM}}$ TGF $\left.\beta\right)^{1}$.

After initial characterization, we observed that all eight primary humanized 4A11 variants preserved the same selectivity profiles as parental antibody (rbt4A11) by binding to human mature TGF $\beta 2$ and TGF $\beta 3$, but not TGF $\beta 1$ (Table 1). Although the humanized variants differed slightly in binding affinity compared to rbt4A11, they retained picomolar binding affinity. However, dramatic differences of blocking activity in the cell-based assay for these humanized variants were observed, which was unexpected because the variants contained almost identical CDRs and closely matched germline frameworks. After detailed analysis, we concluded that humanized variants ( $v 3,4,7$, and 8 ) using the heavy chain germline (IGHV4-59*06) as an acceptor framework maintained similar potency compared to rbt4A11, whereas the humanized variants ( $(1,2,5$ and 6$)$ using the other heavy chain germline (IGHV3-48*01) significantly lost their blocking activity and also turned into incomplete blockers without reaching maximum inhibition at the highest antibody concentration $(<70 \%)$. The usage of different light chain germlines had minimal impact on the blocking activity. Among all eight primary humanized variants, $v 7$ had the most desirable

affinity (TGFB2_K $\left.K_{D}: 6.3 p M ; T G F \beta 3 \_K_{D}: 9.7 p M\right)$ with the best blocking activity (TGFß2_IC 50 : 0.9nM; TGF $\left.33 \_I C_{50}: 0.2 \mathrm{nM}\right)$, and was pursued further as the lead candidate ${ }^{1}$. This result pointed out that the usage of two different yet closely related human heavy chain germlines in the humanization led to the dramatic difference in their blocking activities.

\section{Crystal structures of TGFB2 in complex with antigen-binding fragments (Fabs) of h4A11.v2 and h4A11.v7}

To further investigate how the heavy chain germline acceptor framework differences in the humanized 4A11 variants resulted in significant functional differences in blocking TGF 32 and TGF 33 , we selected one of the incomplete blockers (h4A11.v2) and the top complete blocker (h4A11.v7) to obtain crystal structures of Fab fragments in complex with TGF $\beta 2$. We reported the crystal structure of the h4A11.v7 Fab in complex with TGF $\beta 2$ recently ${ }^{1}$. To investigate any structural differences in the TGF $\beta 2: F a b$ complexes for both v2 and v7 variants, we crystallized and solved the x-ray crystal structure of TGF 32 in a complex with Fab from h4A11.v2 to $2.9 \AA$ resolution (Table 2). The overall structures of both $v 7$ and v2 complexes are very similar, with two Fab fragments binding to two TGF 32 molecules and 
the epitope of each Fab containing contributions from both TGF 32 monomers in the dimer for the h4A11.v2-Fab, as previously reported for the h4A11.v7-Fab (Figure 2). The overall RMSD for all atoms in TGF $\beta 2$ and the variable regions (Fv) of the complexes is $1.04 \AA$. The constant regions of the Fabs were excluded from these calculations because the wide variation in elbow angle of Fabs distorts the overall alignments in an irrelevant way ${ }^{7}$.

An analysis of TGF $\beta 2: h 4 A 11 . v 2-F a b$ and TGF $\beta 2: h 4 A 11 . v 7-F a b$ crystal structures revealed that the epitopes of both h4A11.v2 and h4A11.v7 antibodies on the TGFB2 dimer are identical (Figure 3). This, along with identical CDRs between the two variants, suggested that the functional differences observed between the two variants cannot be explained by a potentially altered epitope. Further analysis of the two structures revealed an intriguing interaction of the 2 heavy chain molecules in the TGF $\beta 2$ :h4A11.v2-Fab complex with each other, which was not present in the TGFß2:h4A11.v7-Fab complex. In the TGF 32 :h4A11.v2-Fab complex structure, Y79, R19 from one heavy chain molecule form a ternary $\pi-$ stacking interaction with Q81 from the other heavy chain molecule and vice versa, resulting in a pseudosymmetric interaction (Figure 4A), which likely orients the relative positions of the two heavy chains and the conformation of the Fabs relative to TGF $\beta 2$ and alters the dynamics of the h4A11.v2 Fab when bound to TGF $\beta 2$ in solution, compared to the h4A11.v7 Fab. Supporting this hypothesis, in the TGF $\beta 2$ :h4A11.v7Fab complex structure, the corresponding residues in the heavy chain framework region are S19, S79 and K81, which do not allow for the specific interactions observed in the TGF $\beta 2$ :h4A11.v2-Fab complex (Figure 4B). Although the distances between the $\mathrm{Ca}$ atoms of the respective residues at each position (19$19,79-79,81-81$ ) in the heavy chains of both the h4A11.v2 and h4A11.v7 Fabs are almost identical in the two structures, we hypothesized that in solution, the interactions between the side chains at 19, 79 and 81 among the 2 heavy chain molecules and subtle effects on the conformations of the Fabs may be the difference between a functional 'complete blocker' and an 'incomplete blocker', where the slight differences induced in the dynamics of the TGF 32 dimer itself due to the presence or absence of the subtle Fab-Fab interaction could play a role. For such an interaction, we assume that two Fabs from the same antibody molecule would bind to a TGF 32 dimer in solution since the rbt4A11 antibodies have monovalent interactions with dimeric TGF $\beta 2$ and TGF $\beta 3$. To test this hypothesis, we performed step by step mutagenesis to try and convert a v2-like function (incomplete blocking) to a v7-like function (complete blocking) with an increasing number of mutations listed in Table 3. We hypothesized that a simple R19S substitution in h4A11.v2 would disrupt the $\pi$-stacking interaction, and so would Q81N or Q81A. In addition, to convert a v7-like function to a v2-like function, we hypothesized that we would need to introduce a greater number of mutations at positions $19,79,81$ to re-build the stabilizing interactions of a v2-like molecule. In this process, we also included an aromatic sidechain $\mathrm{W}$ at position 79, as it could result in a $\pi$-stacking interaction similar to $Y$ in the TGFß2:h4A11.v2-Fab structure (Figure 4).

\section{Generation and functional characterization of structure-guided h4A11.v2 and h4A11.v7 heavy chain framework variants}

To test the hypothesis in accordance with our structural analysis, a new panel of variants was generated with single or multiple mutations at position 19 (HC-FWR1), 79 and 81 (HC-FWR3) of h4A11.v2 
and h4A11.v7. Following expression and purification, all variants were fully characterized and compared with parental rbt4A11, h4A11.v2 and h4A11.v7. As predicted from our structural analysis, all h4A11.v2based variants (h4A11.v2.1 - h4A11.v2.6) demonstrated binding affinity improvement as well as regained complete blocking activity in the cell-based assay against TGF 32 and TGF 33 (Table 3). All three single mutation variants (v2.1_R19S; v2.2_Q81N; v2.3_Q81A) were sufficient to restore complete blocking function similar to rbt4A11 (v2.1 in Figure 5). Furthermore, by adding other mutations to R19S (v2.4_ R19S, Y79S; v2.5_R19S, Q81S; v2.6_R19S, Y79S, Q81S), did not further change the effect of the individual mutations, suggesting the critical role of positions 19,79 and 81 in the observed h4A11.v2 heavy-chain interactions upon TGF 32 and TGF 33 binding. In contrast, all h4A11.v7-based variants (h4A11.v7.1 - h4A11.v7.6) with double mutations (v7.1_S19R, S79Y; v7.2_S19R, S79W) and triple mutations (v7.3_S19R, S79Y, K81Q; v7.4_S19R, S79Y, K81R; v7.5_S19R, S79W, K81Q; v7.6_S19R, S79W, $\mathrm{K} 81 \mathrm{R}$ ) appeared to have a broad range of affinity drop (4-34 fold) compared to rbt4A11 and turned into incomplete blockers against TGF $\beta 2$ and TGF $\beta 3$ (v7.1 in Figure 5). These results supported the important functional role of short sidechain amino acids at human framework positions 19 and 79 in the active h4A11.v7 molecule.

\section{Discussion}

In our current work, we identified a unique and interesting phenomenon during humanization of an allosteric antibody against TGF $\beta 2$ and TGF $\beta 3$, where a single amino acid in the framework region regulates antibody function. This work highlights how subtle differences in framework regions with no effect on antigen binding can dramatically alter antibody function in allosteric ligand blocking. We also demonstrate that it is possible to convert the inhibitory properties of the humanized antibodies by a single amino acid change in framework.

Functional antibodies impart their effects on their targets primarily through the interaction of their CDRs with the antigen. For symmetric, multimeric antigens, where appropriate relative orientations of each monomer are important for binding to partner proteins and for signaling, the existence of multiple epitopes of the antibody (Fab) and the multimeric nature of the interaction between antigens and Fabs could have consequences for signaling. This may offer clues to further design favorable properties for antibodies by varying the regions of antibodies beyond CDRs. As illustrated in this report, the interactions (or lack thereof) between the heavy chain framework regions in two Fabs can have a profound impact on signaling.

Although humanization is a well-established art in the field of antibody engineering, potential learnings especially from allosteric antibodies against antigens that function as multimers could be useful in improved antibody design. In this work, we have introduced and demonstrated an effective approach to humanize the rabbit monoclonal antibody 4A11 using the combinations of CDR grafts and framework shuffling. This approach has been successful in our humanization of multiple rabbit mAbs (data not shown). During the humanization process, we usually select the variants with activities similar to parental antibodies to move forward. However, in the humanization of $4 \mathrm{~A} 11$, the variants displayed 
intriguing properties with similar binding affinity, but dramatically different blocking activity in cell based activity assays. The crystal structures of h4A11.v2 and h4A11.v7 Fabs bound to TGF $\beta 2$ have further delineated the mechanism of the allosteric inhibition that is dependent on homotypic interactions between framework regions of heavy chains. The subtle difference in the human framework impacts the interactions between the heavy chains framework regions of the two Fabs of humanized 4A11 that leads to the loss of activity in h4A11.v2, but not h4A11.v7.

Interaction between framework regions of the heavy chain of Fabs within a functional antibody has been observed before, for example, the anti-HIV-gp120 neutralizing Fab, 2G12 which was isolated from a patient that was resistant to several strains of $\mathrm{HIV}^{8}$. In the case of $2 \mathrm{G} 12$, the variable domain of one heavy chain undergoes a domain exchange with the variable domain of the 2 nd light chain in the antibody and vice versa resulting in a dimerized Fab ${ }^{9}$. In addition, in 2G12, the typically surface exposed framework regions of the heavy chain are also extensively modified to form a specific stable interaction between the framework regions of the two heavy chains. This places the paratopes of the antibody in a defined, rigid geometry, that allows specific recognition of the carbohydrate clusters on the HIV gp120, which would not be possible with a typical IgG scaffold, where there are no stabilizing interactions between the heavy chain variable domains.

Our work, in addition to the $2 \mathrm{G} 12$ example above, suggests that the framework regions offer yet another region in an antibody for engineering interactions between Fabs and modulating antibody function. This could especially be useful where antigens are multimeric and restricting the paratopes on Fabs in a defined orientation to match a geometrically restricted and repeated epitope in a multimeric target could be useful to impart agonistic or antagonistic properties to the antibody, on top of binding properties imparted by CDRs, based on the molecular mechanism of signaling of the antigen and its effector molecules.

\section{Declarations}

\section{Acknowledgements}

We thank Tianhe Sun, Joe Arron, Ryan Kelly and Matthieu Masureel for discussions and suggestions, LMDD rabbit discovery, molecule biology and protein production groups for the support.

\section{Author Contributions}

WCL, PL, JS and YW designed the research, interpreted the data and wrote the paper. WCL, JY, JZ and $\mathrm{KML}$ performed the experiments.

\section{Funding details}

All authors are employees at Genentech Inc. 
All authors are Roche stock holders. No potential conflict of interest was disclosed.

\section{References}

1. Sun, T. et al. TGF 32 and TGF $\beta 3$ isoforms drive fibrotic disease pathogenesis. Sci Trans/ Med, 13, https://doi.org/10.1126/scitranslmed.abe0407 (2021).

2. Jones, P. T., Dear, P. H., Foote, J., Neuberger, M. S. \& Winter, G. Replacing the complementaritydetermining regions in a human antibody with those from a mouse. Nature, 321, 522-525 https://doi.org/10.1038/321522a0 (1986).

3. Foote, J. \& Winter, G. Antibody framework residues affecting the conformation of the hypervariable loops. J Mol Biol, 224, 487-499 https://doi.org/10.1016/0022-2836(92)91010-m (1992).

4. Dall'Acqua, W. F. et al. Antibody humanization by framework shuffling., 36, 43-60 https://doi.org/10.1016/j.ymeth.2005.01.005 (2005).

5. Damschroder, M. M. et al. Framework shuffling of antibodies to reduce immunogenicity and manipulate functional and biophysical properties. Mol Immunol, 44, 3049-3060 https://doi.org/10.1016/j.molimm.2006.12.019 (2007).

6. Dondelinger, M. et al. Understanding the Significance and Implications of Antibody Numbering and Antigen-Binding Surface/Residue Definition. Front Immunol, 9, 2278 https://doi.org/10.3389/fimmu.2018.02278 (2018).

7. Stanfield, R. L., Zemla, A., Wilson, I. A. \& Rupp, B. Antibody elbow angles are influenced by their light chain class. J Mol Biol, 357, 1566-1574 https://doi.org/10.1016/j.jmb.2006.01.023 (2006).

8. Trkola, A. et al. Human monoclonal antibody $2 \mathrm{G} 12$ defines a distinctive neutralization epitope on the gp120 glycoprotein of human immunodeficiency virus type $1 . J$ Virol, 70, 1100-1108 https://doi.org/10.1128/JVI.70.2.1100-1108.1996 (1996).

9. Calarese, D. A. et al. Antibody domain exchange is an immunological solution to carbohydrate cluster recognition., 300, 2065-2071 https://doi.org/10.1126/science.1083182 (2003).

10. Lefranc, M. P. et al. IMGT, the international ImMunoGeneTics database. Nucleic Acids Res, 27, 209212 https://doi.org/10.1093/nar/27.1.209 (1999).

11. Bos, A. B. et al. Optimization and automation of an end-to-end high throughput microscale transient protein production process. Biotechnol Bioeng, 112, 1832-1842 https://doi.org/10.1002/bit.25601 (2015).

12. Luan, P. et al. Automated high throughput microscale antibody purification workflows for accelerating antibody discovery. MAbs, 10, 624-635 https://doi.org/10.1080/19420862.2018.1445450 (2018).

13. Zou, Z. \& Sun, P. D. An improved recombinant mammalian cell expression system for human transforming growth factor-beta2 and -beta3 preparations. Protein Expr Purif, 50, 9-17 https://doi.org/10.1016/j.pep.2006.06.022 (2006). 
14. Otwinowski, Z. \& Minor, W. Processing of X-ray diffraction data collected in oscillation mode. Methods Enzymol, 276, 307-326 (1997).

15. McCoy, A. J., Grosse-Kunstleve, R. W., Storoni, L. C. \& Read, R. J. Likelihood-enhanced fast translation functions. Acta Crystallogr D Biol Crystallogr, 61, 458-464 https://doi.org/10.1107/S0907444905001617 (2005).

16. Del Amo-Maestro, L., Marino-Puertas, L., Goulas, T. \& Gomis-Rüth, F. X. Recombinant production, purification, crystallization, and structure analysis of human transforming growth factor $\beta 2$ in a new conformation. Sci Rep, 9, 8660 https://doi.org/10.1038/s41598-019-44943-4 (2019).

17. Adams, P. D. et al. PHENIX: a comprehensive Python-based system for macromolecular structure solution. Acta Crystallogr D Biol Crystallogr, 66, 213-221

https://doi.org/10.1107/S0907444909052925 (2010).

18. Emsley, P. \& Cowtan, K. Coot: model-building tools for molecular graphics. Acta Crystallogr D Biol Crystallogr, 60, 2126-2132 https://doi.org/10.1107/S0907444904019158 (2004).

\section{Tables}

Table 1. Functional characterization of primary humanized 4A11 variants.

\begin{tabular}{|c|c|c|c|c|c|c|c|c|c|}
\hline \multirow{2}{*}{ Variants } & \multicolumn{2}{|c|}{$\begin{array}{l}\text { Human germline acceptor } \\
\text { framework }\end{array}$} & \multicolumn{3}{|c|}{$\begin{array}{c}\text { TGF } \beta \text { binding by } \\
\text { Biacore SPR (KD: } \mathrm{pM})\end{array}$} & \multicolumn{3}{|c|}{$\begin{array}{c}\text { TGF } \beta \text { inhibition by } \\
\left.\text { HEK-Blue cells ( } \mathrm{IC}_{50}: \mathrm{nM}\right)\end{array}$} & \multirow{2}{*}{ 'Blocking } \\
\hline & $\mathrm{LC}$ & $\mathrm{HC}$ & TGF $\beta 1$ & TGF $\beta 2$ & TGF $\beta 3$ & TGF $\beta 1$ & TGF $\beta 2$ & TGF $\beta 3$ & \\
\hline rbt4A11 & - & - & $>5000^{\mathrm{a}}$ & 1.6 & 1.2 & $>667^{b}$ & 0.9 & 0.1 & Complete \\
\hline h4A11.v1 & IGKV4-1*01 & IGHV3-48*01 & $>5000^{\mathrm{a}}$ & 4.6 & 14.4 & $>667^{b}$ & $>667^{b}$ & $>667^{\mathrm{b}}$ & Incomplete \\
\hline h4A11.v2 & IGKV1D-39*01 & IGHV3-48*01 & $>5000^{\mathrm{a}}$ & 1.4 & 13.3 & $>667^{b}$ & $>667^{b}$ & $>667^{\mathrm{b}}$ & Incomplete \\
\hline h4A11.v3 & IGKV4-1*01 & IGHV4-59*06 & $>5000^{\mathrm{a}}$ & 9.6 & 12.1 & $>667^{b}$ & 3.9 & 0.2 & Complete \\
\hline h4A11.v4 & IGKV1D-39*01 & IGHV4-59*06 & $>5000^{\mathrm{a}}$ & 10 & 10.1 & $>667^{b}$ & 1.9 & 0.1 & Complete \\
\hline h4A11.v5 & IGKV3-20*01 & IGHV3-48*01 & $>5000^{\mathrm{a}}$ & 12.1 & 13.9 & $>667^{b}$ & $>667^{b}$ & $>667^{b}$ & Incomplete \\
\hline h4A11.v6 & IGKV2-24*01 & IGHV3-48*01 & $>5000^{\mathrm{a}}$ & 8.4 & 12.5 & $>667^{b}$ & $>667^{b}$ & $>667^{b}$ & Incomplete \\
\hline h4A11.v7 & IGKV3-20*01 & IGHV4-59*06 & $>5000^{\mathrm{a}}$ & 6.3 & 9.7 & $>667^{b}$ & 0.9 & 0.2 & Complete \\
\hline h4A11.v8 & IGKV2-24*01 & IGHV4-59*06 & $>5000^{\mathrm{a}}$ & 8.2 & 14.1 & $>667^{b}$ & 4.8 & 0.7 & Complete \\
\hline
\end{tabular}

aThe binding was not observed at the highest TGF 11 concentration ( $5 \mathrm{nM})$; therefore, the reported KD are lower limits.

${ }^{b}$ The maximun inhibition did not achieve over $90 \%$ at the highest antibody concentration (667nM); therefore, the reported IC50 are lower limits.

'The complete blocking or incomplete blocking was defined by TGF $\beta 2$ and TGF $\beta 3$ inhibition greater than $90 \%$ or less than $70 \%$, respectively, at the highest antibody concentration.

Table 2. Data collection and refinement statistics. 


\begin{tabular}{|c|c|}
\hline & $\begin{array}{c}\text { TGFß2:4A11.V2-Fab } \\
\text { complex }\end{array}$ \\
\hline \multicolumn{2}{|l|}{ Data collection } \\
\hline Space group & P 22121 \\
\hline \multicolumn{2}{|l|}{ Cell dimensions } \\
\hline a, b, c $(\AA)$ & $60.15,103.48,196.25$ \\
\hline $\mathrm{a}, \mathrm{b}, \mathrm{g}\left({ }^{\circ}\right)$ & $90,90,90$ \\
\hline Resolution ( $\AA)$ & $45.95-2.90(3.08-2.90)^{*}$ \\
\hline $\mathrm{R}_{\text {pim }}$ & $0.021(0.389)$ \\
\hline $\mathrm{I} / \sigma \mathrm{I}$ & $11.6(1.34)$ \\
\hline Completeness (\%) & $98.50(99.70)$ \\
\hline Redundancy & $4.0(4.3)$ \\
\hline $\mathrm{CC}_{1 / 2}$ & $0.998(0.663)$ \\
\hline \multicolumn{2}{|l|}{ Refinement } \\
\hline Resolution ( $\AA)$ & 2.90 \\
\hline No. reflections & $27582(2630)$ \\
\hline $\mathrm{R}_{\text {work }} / \mathrm{R}_{\text {free }}$ & $\begin{array}{c}0.2080(0.3217) / 0.2860 \\
(0.3482)\end{array}$ \\
\hline No. atoms & 8200 \\
\hline Protein & 8152 \\
\hline Ligand/ion & 0 \\
\hline Water & 48 \\
\hline Average $B$-factors & 77.40 \\
\hline Protein & 77.40 \\
\hline Ligand/ion & 0.00 \\
\hline Water & 64.90 \\
\hline \multicolumn{2}{|l|}{ R.m.s. deviations } \\
\hline Bond lengths ( $\AA$ ) & 0.013 \\
\hline Bond angles $\left({ }^{\circ}\right)$ & 1.229 \\
\hline
\end{tabular}

*Values in parentheses are for highest-resolution shell.

Table 3. Functional characterization of humanized $4 \mathrm{~A} 11$ version 2 and version 7 heavy chain framework mutation variants. 


\begin{tabular}{|c|c|c|c|c|c|c|c|}
\hline \multirow[t]{2}{*}{ Variants } & \multirow[t]{2}{*}{ Mutation } & \multicolumn{3}{|c|}{$\begin{array}{c}\text { TGF } \beta \text { binding by } \\
\text { Biacore SPR (KD: } \mathrm{pM})\end{array}$} & \multicolumn{2}{|c|}{$\begin{array}{c}\text { TGF } \beta \text { inhibition by } \\
\text { HEK-Blue cells }\left(\mathrm{IC}_{50}: \mathrm{nM}\right)\end{array}$} & \multirow[t]{2}{*}{ 'Blocking } \\
\hline & & TGF $\beta 1$ & TGF $\beta 2$ & TGF $\beta 3$ & TGF $\beta 2$ & TGF $\beta 3$ & \\
\hline h4A11.v2.1 & R19S & $>5000^{\mathrm{a}}$ & 2.2 & 0.6 & 0.7 & 0.1 & Complete \\
\hline h4A11.v2.2 & Q81N & $>5000^{\mathrm{a}}$ & 4.5 & 6.0 & 2.2 & 0.4 & Complete \\
\hline h4A11.v2.3 & Q81A & $>5000^{\mathrm{a}}$ & 3.4 & 5.5 & 1.7 & 0.7 & Complete \\
\hline h4A11.v2.4 & R19S, Y79S & $>5000^{\mathrm{a}}$ & 1.4 & 0.6 & 2.2 & 0.2 & Complete \\
\hline h4A11.v2.5 & R19S, Q81S & $>5000^{\mathrm{a}}$ & 1.7 & 0.6 & 0.7 & 0.1 & Complete \\
\hline h4A11.v2.6 & R19S, Y79S, Q81S & $>5000^{\mathrm{a}}$ & 1.9 & 0.5 & 1.7 & 0.3 & Complete \\
\hline h4A11.v7.1 & S19R, S79Y & $>5000^{\mathrm{a}}$ & 10.7 & 40.5 & $>667^{b}$ & $>667^{b}$ & Incomplete \\
\hline h4A11.v7.2 & S19R, S79W & $>5000^{\mathrm{a}}$ & 9.6 & 22.8 & $>667^{b}$ & $>667^{b}$ & Incomplete \\
\hline h4A11.v7.3 & S19R, S79Y, K81Q & $>5000^{\mathrm{a}}$ & 5.7 & 8.0 & $>667^{b}$ & $>667^{b}$ & Incomplete \\
\hline h4A11.v7.4 & S19R, S79Y, K81R & $>5000^{\mathrm{a}}$ & 11.2 & 18.8 & $>667^{b}$ & $>667^{b}$ & Incomplete \\
\hline h4A11.v7.5 & S19R, S79W, K81Q & $>5000^{\mathrm{a}}$ & 5.6 & 7.2 & $>667^{b}$ & $>667^{b}$ & Incomplete \\
\hline h4A11.v7.6 & S19R, S79W, K81R & $>5000^{\mathrm{a}}$ & 9.7 & 15.6 & $>667^{\mathrm{b}}$ & $>667^{\mathrm{b}}$ & Incomplete \\
\hline
\end{tabular}

${ }^{a}$ The binding was not observed at the highest TGF $\beta 1$ concentration ( $\left.5 \mathrm{nM}\right)$; therefore, the reported KD are lower limits.

${ }^{b}$ The maximun inhibition did not achieve over $90 \%$ at the highest antibody concentration (667nM); therefore, the reported IC50 are lower limits.

'The complete blocking or incomplete blocking was defined by TGF $\beta 2$ and TGF $\beta 3$ inhibition greater than $90 \%$ or less than $70 \%$, respectively, at the highest antibody concentration.

Figures

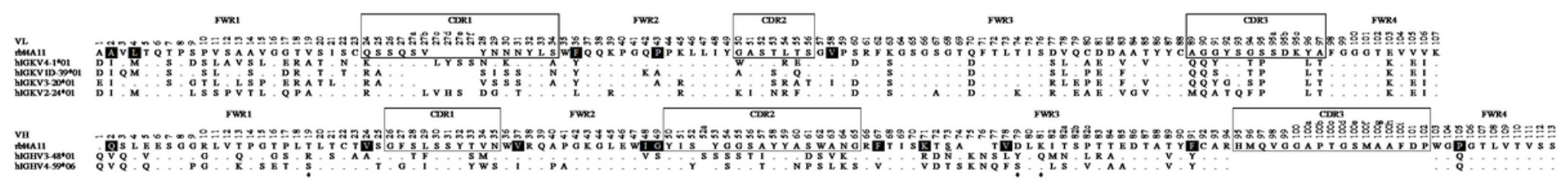

Figure 1

Amino acid sequence alignment of $\mathrm{VL}$ and $\mathrm{VH}$ of rbt4A11 with the closest human germline acceptor frameworks for humanization. The variable light chain $(\mathrm{VL})$ and heavy chain $(\mathrm{VH})$ domain of rbt4A11 was aligned with its closets human germlines to specify the different (letter) and identical (dot) amino acid residues in framework regions (FWR) and complementarity-determining regions (CDRs) by Kabat numbering scheme6. Six CDRs (box) and fifteen framework residues at Vernier zone (black fill) of rbt4A11 were fused in-frame to individual human germline acceptor framework for shuffling. Three positions $(19,79,81)$ at the heavy chain framework guided by structure complex analysis were residues 
used to modulate antibody function (asterisk). The positions with sequence deletion and undefined heavy chain germline CDR3 were shown in blanks.
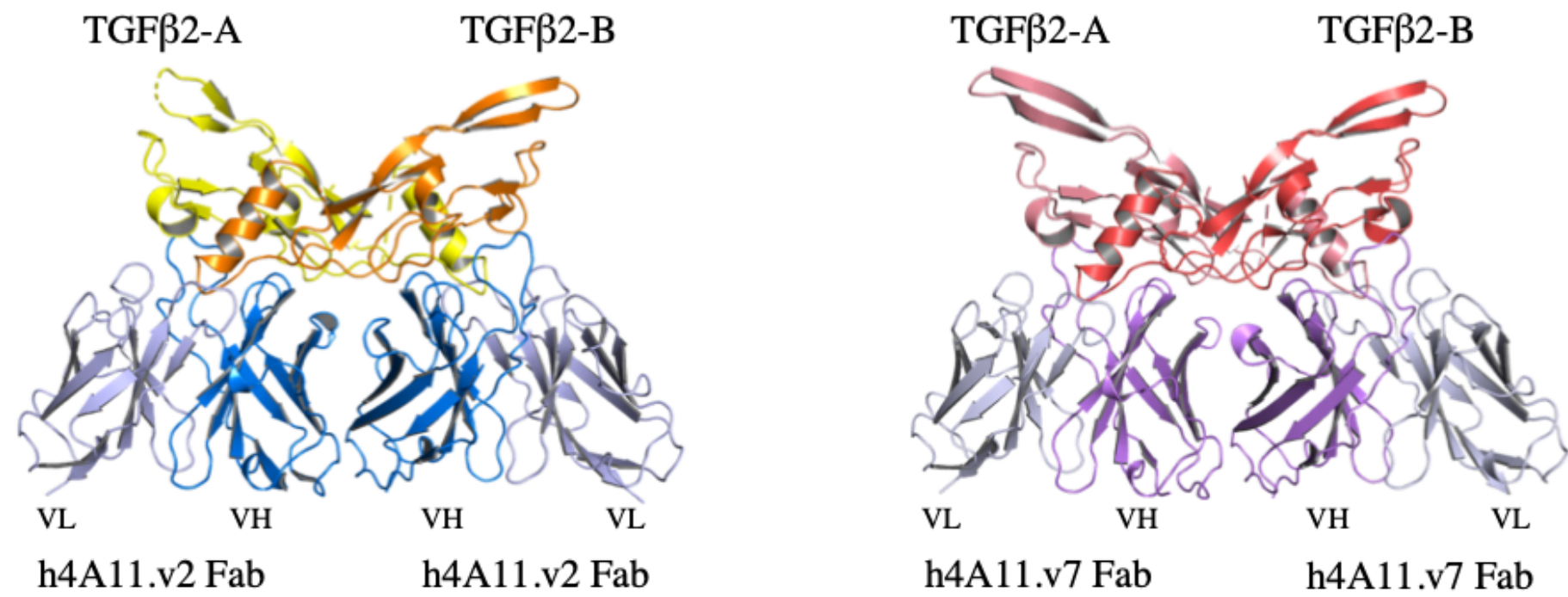

Figure 2

The structure complexes of h4A11.v2 and h4A11.v7 Fab with TGFB2 are similar Overall structures of TGF $\beta 2$ ( $A$ and $B$ chain):h4A11-Fab variant complexes v2 and v7 are very similar with RMSD of $1.04 \AA$ for all atoms (only VH-VL regions in the Fab are shown for clarity).

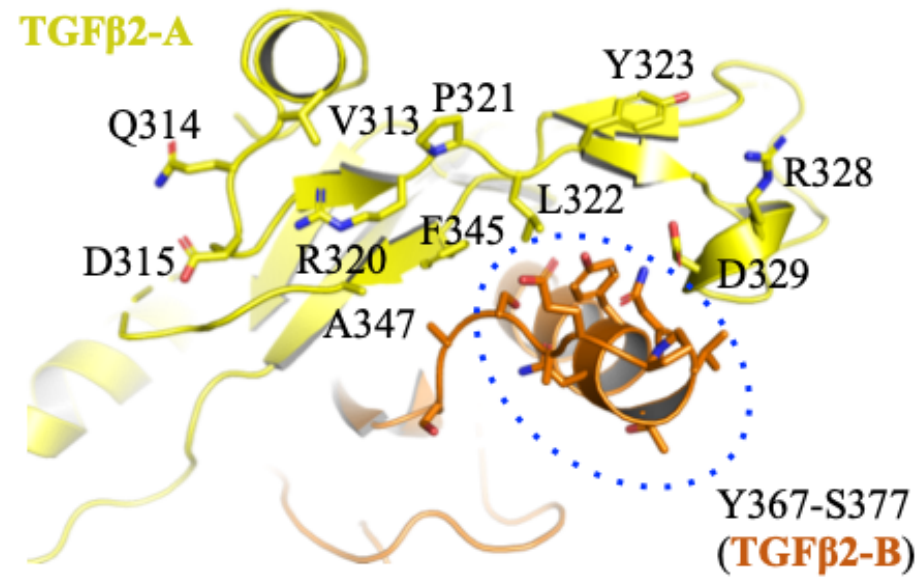

h4A11.v2 epitope on TGF $\beta 2$ dimer

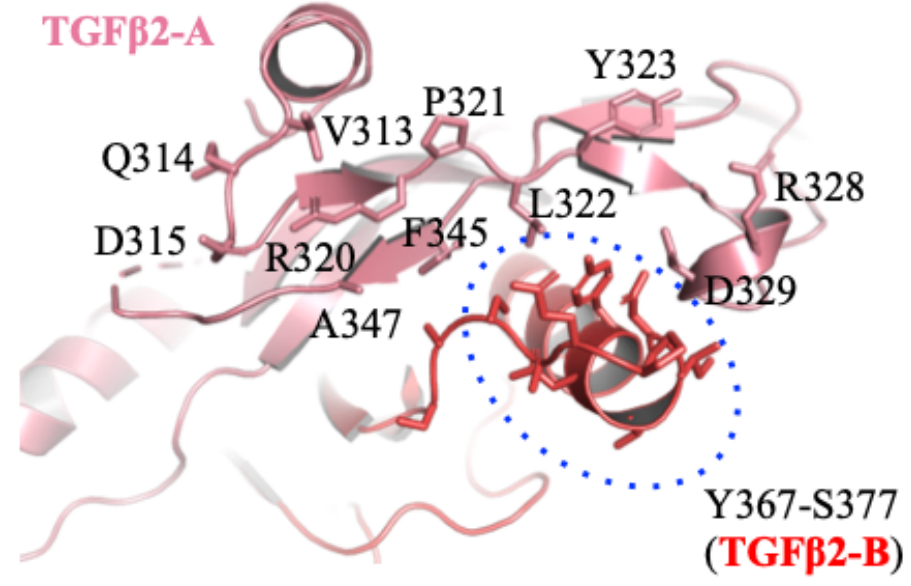

h4A11.v7 epitope on TGF $\beta 2$ dimer

Figure 3

The epitopes of h4A11.v2 and h4A11.v7 on TGF 22 are identical The epitopes of h4A11.v2 and h4A11.v7 are shown and labelled by amino acid with its corresponding position on each monomer of TGF $\beta 2$ ( $A$ and B chain). 
(A) h4A11.v2:TGFß2 Complex

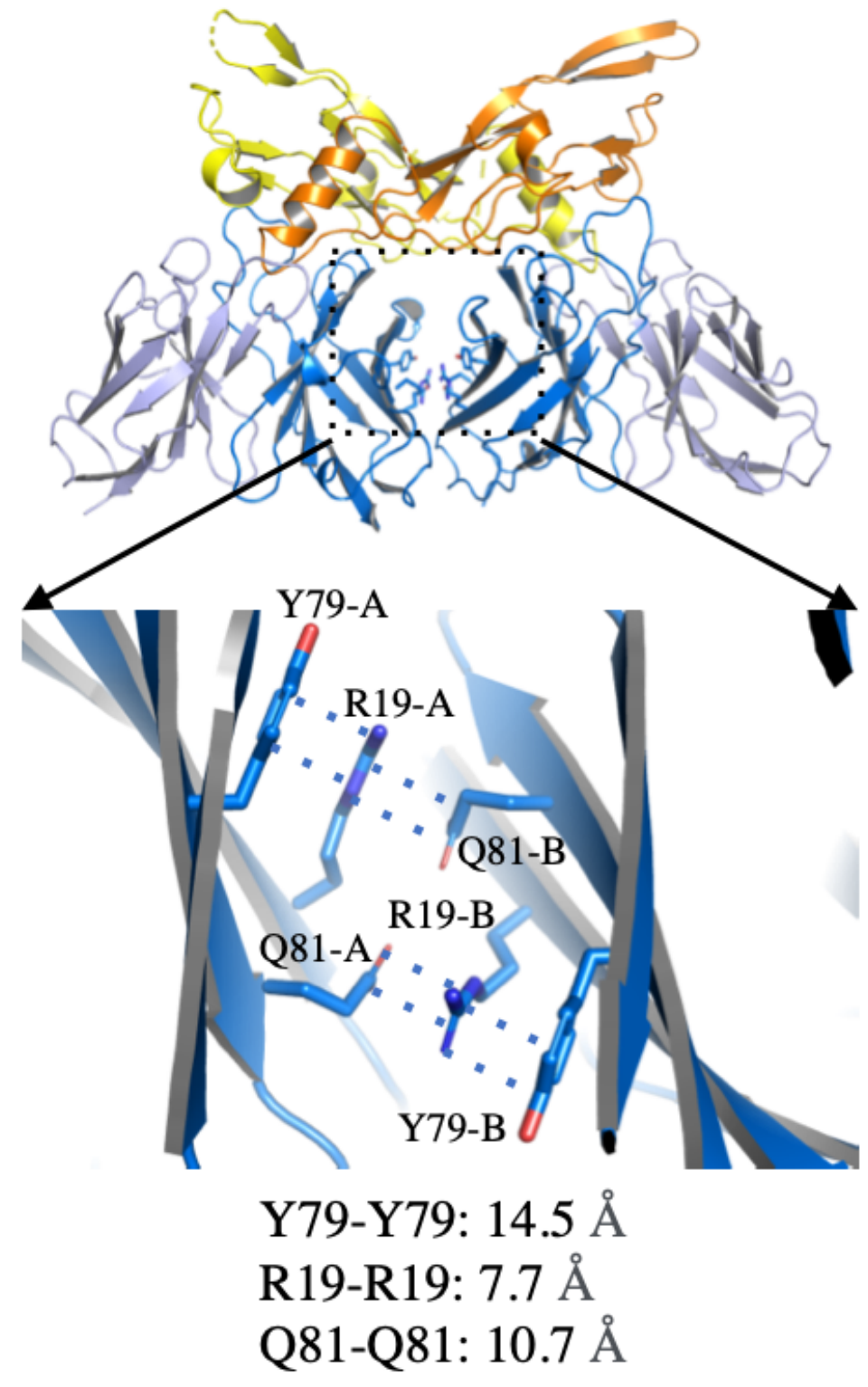

(B) h4A11.v7:TGFß2 Complex

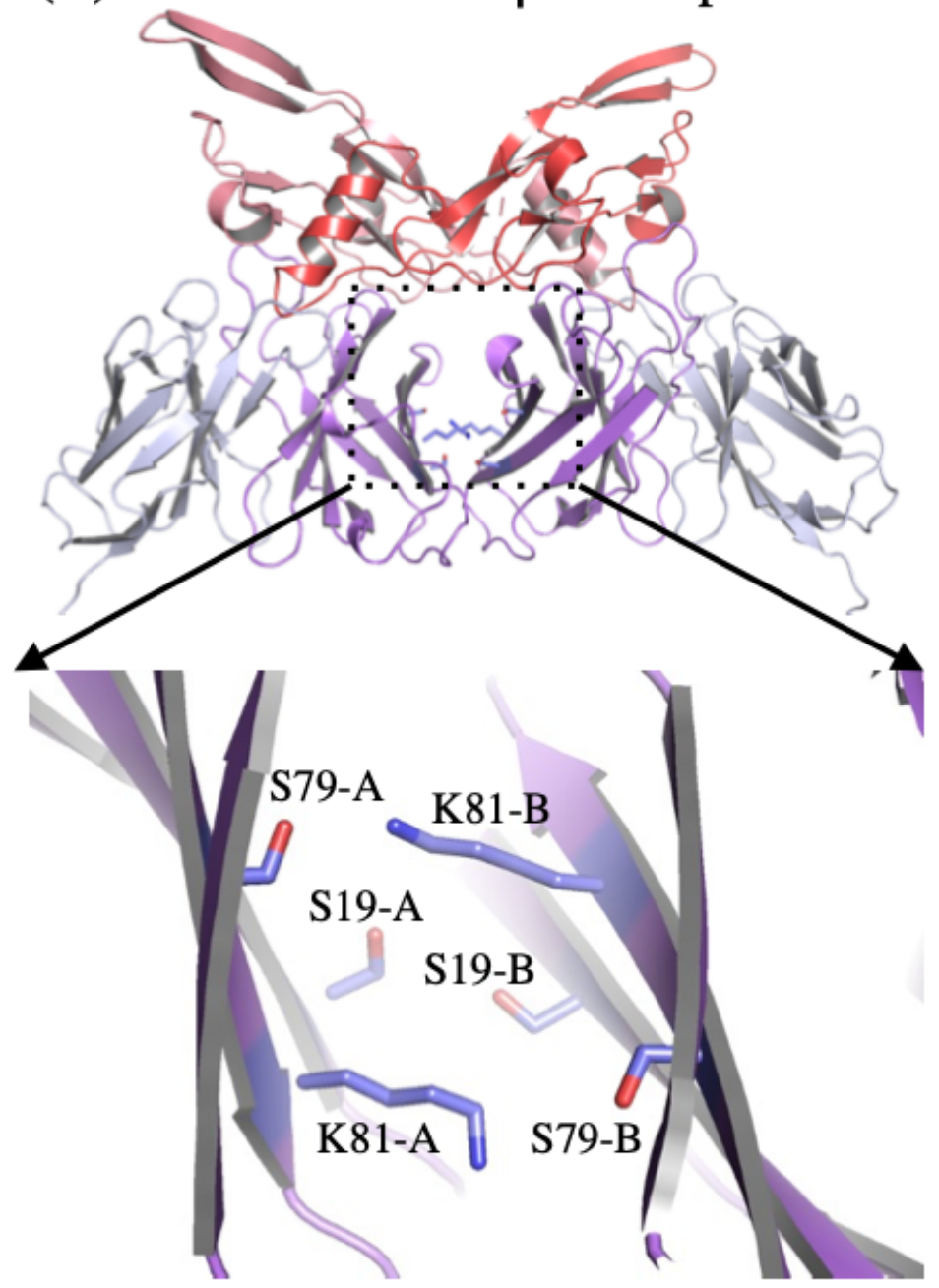

S79-S79: $14.5 \AA$

S19-S19: $7.7 \AA$

K81-K81: $10.5 \AA$

Figure 4

Close view of molecular interaction between 2 Fabs with TGF 32 binding (A) $\pi$-stacking between $Y 79$ and R19 from one heavy chain A and Q81 from the second heavy chain B and vice versa results in a stable interaction between the two heavy chains of the Fabs in the h4A11.v2:TGF 32 complex. (B) Aforementioned $\pi$-stacking is disrupted in h4A11.v7:TGF $\beta 2$ complex because of the substitution in the framework region of the heavy chain residues to S79, S19 and K81. 

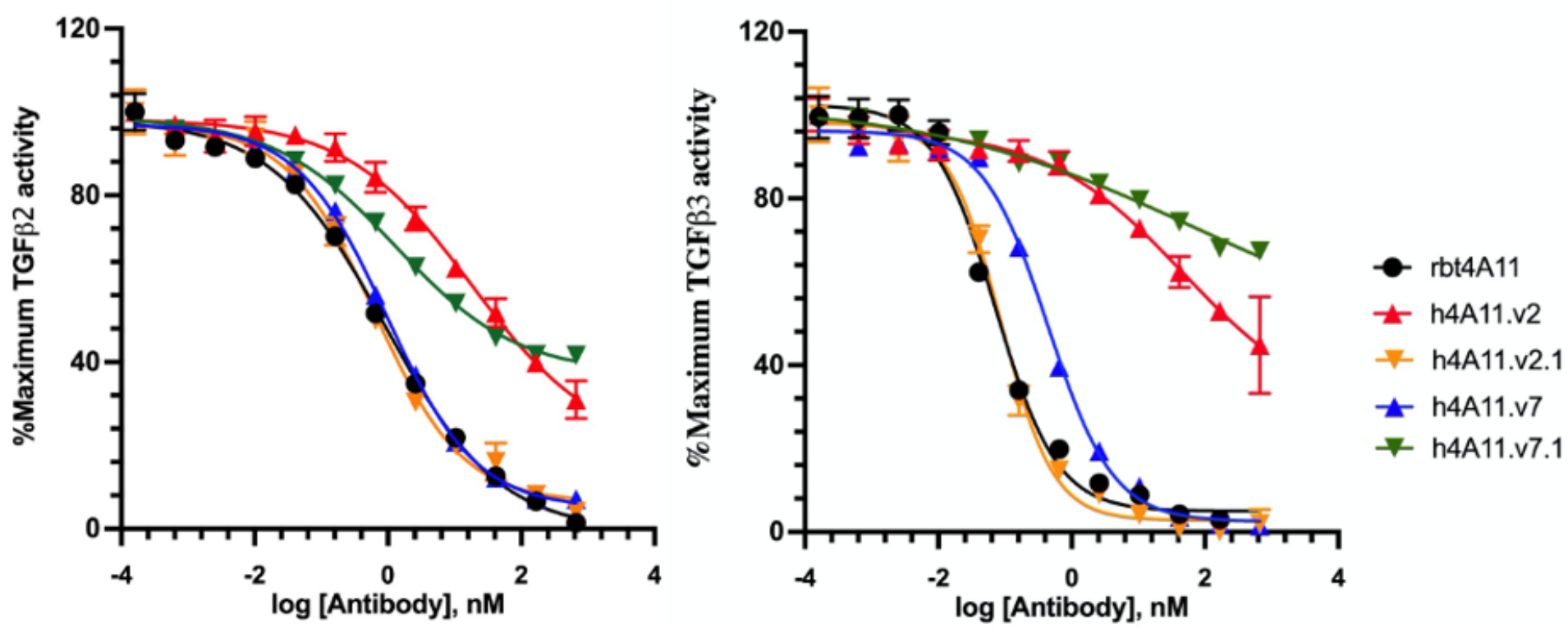

Figure 5

Dose-dependent inhibition of TGF $\beta$ by humanized $4 A 11$ variants in HEK Blue ${ }^{\mathrm{TM}}$ TGF $\beta$ reporter cell-based assay. Human mature TGF $\beta 2$ (left) or TGF $\beta 3$ (right) isoform was incubated with a serial dilution of humanized 4A11 variants in quadruplicate to generate a dose-dependent inhibition curve. The response was normalized to maximum TGF $\beta$ activity (\%) and mean activity + standard deviation was plotted as a function of antibody concentration. The variants exhibiting less than $10 \%$ of maximum TGF $\beta$ activity at the highest antibody concentration were complete blocker (e.g., rbt4A11, h4A11.v2.1, h4A11.v7), whereas those reaching greater than $30 \%$ of maximum TGF $\beta$ activity were incomplete blockers (e.g., h4A11.v2, h4A11.v7.1). 